\title{
Use of haemodialysis plastic cannula with ultrasound guidance in difficult arteriovenous access
}

\section{Dear Editor,}

The blind technique using metal needles has been the traditional method of cannulating arteriovenous (AV) accesses for haemodialysis. While most mature AV accesses can be cannulated with metal needles successfully, the incidence of miscannulation can be high in difficult accesses due to their location and depth, or in new accesses. ${ }^{1}$ Cannulation-related complications were reported to be as high as $31 \%$ and may eventually lead to access failure. ${ }^{2}$ Technical difficulty also increases following a miscannulation as infiltration and haematomas may increase the depth of the AV access. ${ }^{3,4}$

The use of metal dialysis needles for cannulation of AV accesses is the current standard of care in our institution, the Singapore General Hospital, Singapore. Plastic dialysis cannulae were recently introduced in the hospital to help improve cannulation of difficult dialysis accesses under ultrasound guidance. This quality audit aims to report the outcomes of ultrasoundguided cannulation of difficult AV accesses using plastic cannulae.

We performed a retrospective audit of patients referred to the Interventional Nephrology Service for ultrasoundguided cannulation of AV access between September 2017 and March 2019. Referred patients had either failed cannulation by dialysis nurses or were assessed to have a high chance of cannulation failure with the blind technique. This quality audit performed with de-identified data is exempt for review by the Institutional Review Board. Outcome measures were defined as the number of successful cannulations, number of passes needed, proportion of patients that successfully completed the prescribed length of haemodialysis, and blood flow achieved.

Twenty-eight ultrasound-guided plastic cannula placements were performed in 22 patients. The median age was 61.5 years (interquartile range [IQR] 53.874.5), 50\% were women, and majority were of Chinese ethnicity (72.7\%). Most accesses were AV fistulas $(77.3 \%)$. The most common anatomical anastomosis was radiocephalic (50.0\%). Seven accesses (31.8\%) were new and had never been successfully used for dialysis. The median access flows of new and all accesses were $509 \mathrm{~mL} / \mathrm{min}$ (IQR 251-817) and $615 \mathrm{~mL} / \mathrm{min}$ (IQR
515-2200), respectively. Indications for ultrasoundguided cannulation included failed blind cannulation by dialysis nurses (48\%), swollen access (41\%) and new access for trial of cannulation (9\%).

A total of 27 arterial and 28 venous site cannulations were recorded (Table 1). All cannulations were successful without immediate complications. Mean blood flow achieved was $214 \pm 24.4 \mathrm{~mL} / \mathrm{min}$. One patient did not achieve the prescribed blood flow due to extreme negative pressures at the arterial needle.

Among the 22 patients, $11(50 \%)$ required only 1 dialysis session with plastic cannula, with subsequent metal cannulation performed successfully by dialysis nurses. Three patients $(13.6 \%)$ required a second dialysis session with plastic cannula. One patient $(4.5 \%)$ underwent angioplasty after the first session with plastic cannula, and subsequent metal cannulation was successful. Six patients $(27.3 \%)$ underwent 1-2 dialysis sessions using plastic cannulae, and subsequently transited to a tunnelled dialysis catheter as the AV accesses were poorly matured. One patient required multiple plastic cannulations over a 2 -week period to allow resolution of the peri-fistula swelling.

Our audit shows that high cannulation success rates for difficult AV accesses can be achieved with ultrasound-guided cannulation using plastic cannulae. The metal and plastic cannula needles used by our institution are both 16-gauge needles, with V-shaped tips. However, the metal needle (SGD0.47/piece, approximately USD0.35) is $30 \mathrm{~mm}$ in length while the plastic cannula (SGD3.00/piece, approx. USD2.23) is $38 \mathrm{~mm}$. Cannulation of the deeper AV access is therefore possible with the longer plastic cannula. Although longer metal needles are commercially available, leaving longer metal needles in situ for 4-5 hours may induce longer segments of endothelial injury from needle contact, and consequently neointimal hyperplasia and stenosis. ${ }^{5,6}$ In comparison, plastic cannulae have the potential to negotiate tortuous AV accesses, reducing risks of infiltration and access intervention along the cannulation segment. ${ }^{6}$

Our results showed that the use of plastic cannulae prevented temporary dialysis catheter insertions in 16 out of 22 patients. This helped to minimise 
Table 1. Immediate and dialysis outcomes of cannulation using plastic canula according to sites

\begin{tabular}{lll}
\hline Outcomes & & Values, $\mathbf{n}(\mathbf{\%})$ \\
\hline & Arterial site $(\mathbf{n}=\mathbf{2 7})$ & Venous site (n=28) \\
\hline Successful cannulation & $27(100)$ & $28(100)$ \\
\hline Complications during cannulation & Nil & Nil \\
\hline Successful at first pass & $25(93)$ & $25(89)$ \\
\hline Completed prescribed length of dialysis & $27(100)$ & $28(100)$ \\
\hline Achieved blood flow prescribed & $26(96)$ & Not applicable \\
\hline
\end{tabular}

catheter-related risks of bloodstream infections, dysfunction, thrombosis and central vein stenosis, ${ }^{7}$ hence avoiding additional procedural costs and prolonged hospitalisations. Ultrasound-guided plastic cannulation may therefore be a feasible bridging method to reduce emergency placement of dialysis catheters while awaiting resolution of $\mathrm{AV}$ access swelling, or definitive procedures such as angioplasty for salvage of nonmaturing AV fistulas. ${ }^{8}$

Miscannulations of $\mathrm{AV}$ accesses can be distressing for both dialysis nurses and patients. Placement of plastic cannulae may increase the confidence and ease of subsequent metal cannulation by dialysis nurses, through the use of previous cannulation marks and tracks. This would be useful for patients who require early use of fistulas, or whose accesses are less prominent due to overlying subcutaneous tissue. Future training for dialysis nurses could include ultrasoundguided cannulation techniques and the use of plastic cannulae to help reduce miscannulations.

Despite the proven benefits of plastic cannulae over metal needles, its use is hampered by cost. ${ }^{4,9}$ In our institution, the price difference of SGD2.50 (approx. USD1.86) per needle would result in increased annual cost of almost SGD800 (approx. USD594.50) per patient. Plastic cannulae are hence only used in patients who have failed, or are likely to fail metal needle cannulation. We had also routinely administered subcutaneous lignocaine prior to insertions of plastic cannulae, hence patients did not report increased pain compared to metal needle cannulations. There is also a theoretical risk of bloodstream infection from the cannulae, which may be associated with high mortality in dialysis patients. ${ }^{10}$ However, no infections were observed, and the cannulae were removed immediately post-dialysis. No other issues, such as cannula dislodgement or increased bleeding after removal of the plastic cannula, were observed.

Due to the small population size, our observations may not be generalisable to other institutions. There was also no control group comparing insertion of conventional metal needles under ultrasound guidance. In addition, certain data such as access vintage, patency rates and depths of $\mathrm{AV}$ access were not available. These can be included in future studies looking at the utility of plastic cannulae. Ultrasound-guided cannulation with plastic cannulae can be safely performed in difficult $\mathrm{AV}$ accesses with high success rates, may reduce the use of dialysis catheters, and may be used as a bridging approach while awaiting definitive interventions.

This audit was presented in oral presentation form at the 4th meeting of the Asian-Pacific Society of Dialysis Access, 11-12 July 2019.

\section{REFERENCES}

1. National Kidney Foundation. Needles and cannulas for arteriovenous fistula access. 2016. Available at: https://www.kidney.org/sites/default/ files/Fistula\%20Bulletin_0.pdf. Accessed on 30 March 2021.

2. Van Loon MM, Kessels AG, Van der Sande FM, et al. Cannulation and vascular access-related complications in hemodialysis: factors determining successful cannulation. Hemodial Int 2009;13:498-504.

3. Grainer F. Plastic (non-metal) fistula cannula: from concept to practice. Ren Soc Australas J 2014;10:44-6.

4. Parisotto MT, Pelliccia F, Bedenbender-Stoll E, et al. Haemodialysis plastic cannulae - a possible alternative to traditional metal needles? J Vasc Access 2016;17:373-9.

5. Marticorena RM, Dacouris N, Donnelley SM. Randomized pilot study to compare metal needles versus plastic cannulae in the development of complications in hemodialysis access. J Vasc Access 2018;19:272-82.

6. Lee T, Roy-Chaudhury P. Advances and new frontiers in the pathophysiology of venous neointimal hyperplasia and dialysis access stenosis. Adv Chronic Kidney Dis 2009;16:329-38. 
7. Teo BW, Ma V, Xu H, et al. Nephrology Clinical Research Group. Profile of hospitalization and death in the first year after diagnosis of end-stage renal disease in a multi-ethnic Asian population. Ann Acad Med Singap 2010;39:79-87.

8. Tham WP, Burgmans MC, Tan BS, et al. Percutaneous endovascular treatment to salvage non-maturing arteriovenous fistulas in a multiethnic asian population. Ann Acad Med Singap 2017;46:64-71.

9. Smith V, Schoch M. Plastic cannula use in hemodialysis access. J Vasc Access 2016;17:405-10.

10. Xu GG, Yam A, Teoh LC, et al. Epidemiology and management of surgical upper limb infections in patients with end-stage renal failure. Ann Acad Med Singap 2010;39:670-5.
Alvin Ren Kwang Tng, ${ }^{1} M R C P$, Ru Yu Tan, ${ }^{1}{ }_{M R C P}$, Suh Chien Pang, ${ }^{1}{ }_{M R C P}$, Li Choo $\underline{\mathrm{Ng}},{ }^{2}{ }_{M N}$, Wenqian Liang, ${ }^{2} M N$, Chieh Suai $\underline{\text { Tan }},{ }_{F R C P}^{1}$

${ }^{1}$ Department of Renal Medicine, Singapore General Hospital, Singapore ${ }^{2}$ Nursing Division, Singapore General Hospital, Singapore

Correspondence: Dr Alvin Ren Kwang Tng, Department of Renal Medicine, Singapore General Hospital, Academia, Level 3, 20 College Road, Singapore 169856.

Email: alvin.tng.r.k@singhealth.com.sg 